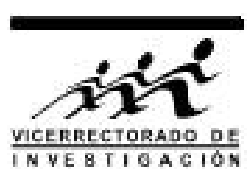

\title{
Cálculo de Parámetros Hipocentrales Mediante el Método de Inversión Generalizada
}

\author{
César Jiménez ${ }^{* 1}$ y Daniel Olcese $^{2}$ \\ ${ }^{1}$ Universidad Nacional Mayor de San Marcos, Ap. Postal 14-0149, Lima, Perú \\ ${ }^{2}$ Dirección de Hidrografía y Navegación DHN
}

Recibido 01 mayo 2017 - Aceptado 20 julio 2017

\begin{abstract}
En este trabajo se aplicó el método de inversión generalizada para calcular los parámetros hipocentrales de eventos sísmicos de origen local y regional a partir de la lectura del tiempo de arribo de las fases $\mathrm{P}$ y $\mathrm{S}$ de al menos 4 estaciones sísmicas. La condición inicial del modelo se obtiene de la posición $\left(x_{0}, y_{0}\right)$ de la estación sísmica más cercana al epicentro; a partir del diagrama de Wadati se obtiene el tiempo origen inicial $t_{0}$ y la profundidad inicial $z_{0}$ se fija en $33 \mathrm{~km}$ para todos los casos. Se asume un modelo de velocidad homogéneo con $v_{p}=7.6 \mathrm{~km} / \mathrm{s}$ (obtenido a partir del proceso de inversión). Del análisis de los autovalores y autovectores de la matriz del sistema $\left(G^{T} G\right)$ se deduce que el parámetro más estable es el tiempo origen y el parámetro más inestable es la profundidad focal. La solución inicial del modelo numérico linealizado converge rápidamente a la solución de menor residual.
\end{abstract}

Palabras claves: sismología, epicentro, inversión.

\section{Calculation of Hypocentral Parameters using the Generalized Inversion Method}

In this research a generalized inversion method was applied to obtain the epicenter of seismic events of local and regional origin from the readings of arrival time of $\mathrm{P}$ and $\mathrm{S}$ phases of at least 4 seismic stations. The initial condition of the model is obtained from the location of the seismic station closest to the epicenter, the initial origin time is obtained from the Wadati diagram and the initial depth is set up to $33 \mathrm{~km}$ for each case. We suppose a homogeneous velocity model of the $P$ wave of $7.6 \mathrm{~km} / \mathrm{s}$ (obtained from the inversion process). From the analysis of eigenvalues and eigenvectors of the system matrix $\left(G^{T} G\right)$, we deduce that the most stable parameter is the origin time and the most unstable parameter is the focal depth. The linearized model converges quickly to the solution.

Keywords: seismology, epicenter, inversion.

\section{Introducción}

La alta sismicidad de la región costera y en general de todo el Perú, se debe a la interacción de las placas tectónicas de Nazca y Sudamericana mediante el proceso de subducción, en la cual la placa de Nazca se introduce debajo de la placa continental, con una velocidad relativa de convergencia entre ambas placas del orden de los 6 a $7 \mathrm{~cm} /$ año (Norabuena et al., 1998). Debido a dicha interacción entre las placas tectónicas se generan esfuerzos y deformaciones de la corteza originando los sismos. A su vez, estos sismos, generan ondas sísmicas, las cuales se propagan a través del medio elástico (ondas de volumen) y a través de la superficie de la Tierra (ondas superficiales). Las ondas de volumen pueden ser de dos tipos: ondas $\mathrm{P}$ o primarias (ondas longitudinales) y ondas $\mathrm{S}$ o secundarias (ondas transversales). Las ondas $\mathrm{P}$ se propagan a mayor velocidad que las ondas $S$.

Una de las rutinas más importantes en la vigilancia y observación sismológica es la localización de la fuente sísmica (y el cálculo de la magnitud sísmica). Esto involucra la determinación de las coordenadas hipocentrales y del tiempo origen. En general, la determinación de la ubicación de la fuente requiere la identificación de las fases sísmicas y la medición de sus tiempos de arribo, así como del conocimiento de la estructura de velocidades entre el hipocentro y la estación sísmica.

Los métodos de localización hipocentral se basan en un modelo de corteza pre-establecido (normalmente se suponen capas planas y paralelas sobre un medio semi-infinito)

\footnotetext{
*cjimenezt@unmsm.edu.pe
} 
y partiendo de un hipocentro inicial de prueba (que suele tener las coordenadas de la estación más cercana incrementadas en una pequeña cantidad), se calculan los tiempos de arribo teóricos y se comparan con los tiempos de arribo observados. Se obtienen así las diferencias entre los tiempos observados y calculados. Las expresiones analíticas de los tiempos de recorrido teóricos son linealizadas y se calculan unos incrementos a las coordenadas hipocentrales y el tiempo origen que minimicen los residuales de todas las observaciones. El método más utilizado para este fin es el de los mínimos cuadrados. El proceso se repite hasta que los incrementos hipocentrales sean lo suficientemente pequeños (Bravo, 1996).

Para determinar los parámetros hipocentrales (latitud y longitud del epicentro, profundidad focal y tiempo origen) se necesitan las lecturas de los tiempos de arribo de las fases $\mathrm{P}$ y $\mathrm{S}$ de 4 estaciones sísmicas como mínimo. Mientras se disponga de datos de más estaciones (con una buena cobertura o distribución azimutal), mejor controlados estarán los parámetros hipocentrales y se obtendrá un mejor resultado.

El problema del cálculo de los parámetros hipocentrales corresponde a un problema inverso, donde los datos están dados por las lecturas de los tiempos de arribo de las ondas sísmicas $\mathrm{P}$ y $\mathrm{S}$ a las estaciones sísmicas. Este problema ha sido resuelto mediante la implementación de algoritmos y códigos computacionales. Uno de los códigos más utilizados es el Hypoinverse (Klein, 2014) y el Hypoellipse (Lahr, 1999).

En este trabajo se ha desarrollado un programa computacional en Matlab para calcular los parámetros hipocentrales mediante el algoritmo o método de inversión generalizada. Como medio de propagación de las ondas sísmicas se ha elegido un medio elástico semi-infinito, isotrópico y homogéneo con velocidad de onda sísmica $\mathrm{P}$ y $\mathrm{S}$ constantes.

\section{Metodología}

\section{Diagrama de Wadati}

El tiempo origen del sismo puede ser determinado mediante una técnica gráfica muy sencilla conocida como diagrama de Wadati. La diferencia de arribo de las ondas sísmicas $\mathrm{S}$ y $\mathrm{P}\left(t_{s}-t_{p}\right)$ es graficada versus el tiempo de arribo de la onda $P$ (Figura 1 ). Puesto que la diferencia de tiempo $t_{s}-t_{p}$ se aproxima a cero en el hipocentro, la recta ajustada sobre el diagrama de Wadati proporciona el tiempo origen aproximado en el intercepto con el eje horizontal de tiempo de arribo de la onda P (Lay y Wallace, 1995).

Asumiendo que la relación de velocidades de las ondas sísmicas $v_{P} / v_{S}$ es constante, la diferencia de tiempo $t_{s}$ - $t_{p}$ puede ser calculada mediante la relación (Havskov, 2010):

$$
t_{s}-t_{p}=\left(\frac{v_{p}}{v_{s}}-1\right)\left(t_{p}-t_{0}\right)
$$

Adicionalmente, a partir de la pendiente de la recta podemos calcular la relación de velocidades $v_{P} / v_{S}$. De la ecuación (1) la pendiente m es: $m=\frac{v_{p}}{v_{s}}-1$, de donde:

$$
\frac{v_{p}}{v_{s}}=m+1
$$

\section{El método directo}

Para el cálculo del epicentro se requiere determinar 4 incógnitas: las 3 coordenadas del hipocentro y el tiempo origen. Como condición inicial, se puede estimar una solución y calcular los tiempos de arribo de la onda P. Si se comparan estos pronósticos con los tiempos observados, se puede determinar el error de la estimación inicial. Luego se corrige la estimación inicial y se repite el proceso hasta obtener una diferencia mínima entre los tiempos de arribo observados y calculados.

El método directo es el procedimiento mediante el cual se generan datos que pueden ser comparados con las observaciones. Cuando se encuentra que un modelo directo se aproxima a los datos observados, se considera que el modelo describe bastante bien la localización epicentral. Matemáticamente, se puede describir en notación matricial:

$$
G_{i j} m_{j}=d_{i}
$$

Donde $G$ es un operador que se aplica al modelo de parámetros $m$ para obtener el vector de datos $d$. El vector $m$ tiene 4 parámetros, para las coordenadas espaciales (longitud, latitud, profundidad) y temporal del epicentro. El vector de datos d contiene la información de los tiempos de arribo a las estaciones sísmicas (Tarantola, 2005).

El método numérico utiliza el ajuste por mínimos cuadrados de las diferencias de tiempo de arribo de las ondas P. El tiempo de arribo $t_{i}$ para $\mathrm{N}$ observaciones de estaciones sísmicas localizadas en $\left(x_{i}, y_{i}\right)$ depende del tiempo origen, latitud, longitud y profundidad focal. Los tiempos de recorrido de las ondas desde el foco a cada estación se deducen por cálculo directo a partir de modelos conocidos de la distribución de la velocidad con la profundidad o en este caso, para un modelo homogéneo de velocidad constante.

Como $t_{i}$ es una función no-lineal se debe linealizar, para ello se realiza un desarrollo en series de Taylor en torno a los valores iniciales aproximados: $\left(t_{0}^{*}, x_{0}, y_{0}, h\right)$, entonces (Udias, 1997):

$$
\begin{gathered}
t_{i}=t_{i}\left(t_{0}^{*}+\delta t, x_{0}+\delta x, y_{0}+\delta y, h+\delta h\right) \\
t_{i}=t_{i}^{*}+\delta t+\frac{\partial t_{i}}{\partial x} \delta x+\frac{\partial t_{i}}{\partial y} \delta y+\frac{\partial t_{i}}{\partial h} \delta h, i=1,2, \ldots, N \\
r_{i}=t_{i}-t_{0}^{*}
\end{gathered}
$$

Donde $r_{i}$ es el residual correspondiente a la solución $\left(t_{0}, x_{0}, y_{0}, h\right), \mathrm{N}$ es el número de datos (estaciones). Las 


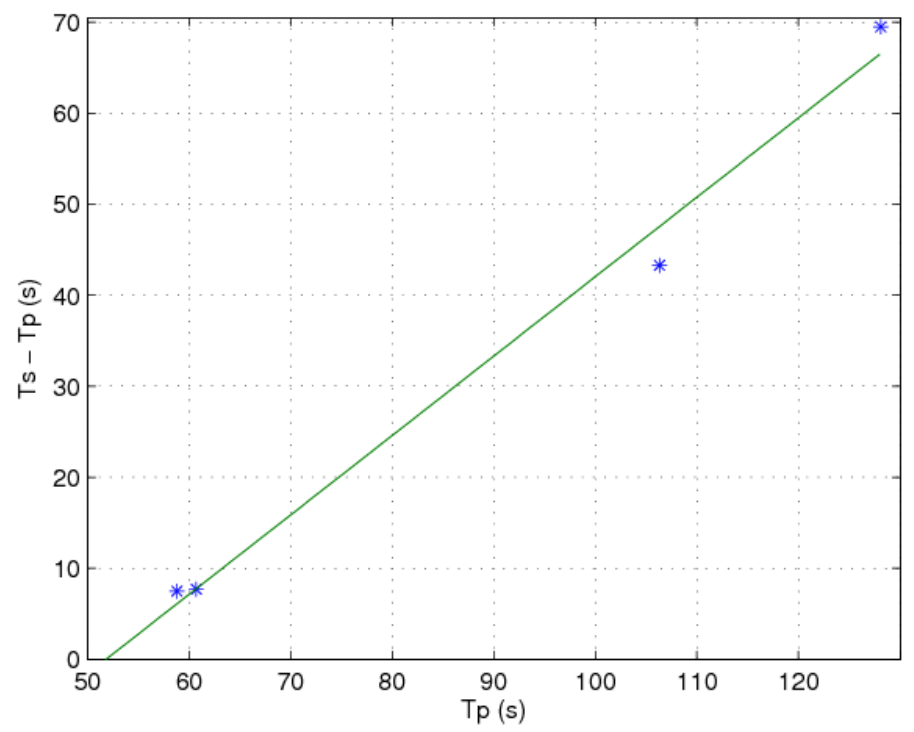

Figura 1: Diagrama de Wadati: $t_{s}-t_{p}$ versus $t_{p}$. El intercepto con el eje horizontal representa el tiempo origen del sismo (51.8 s). La relación entre las velocidades $\mathrm{P}$ y $\mathrm{S}$ es 1.87.

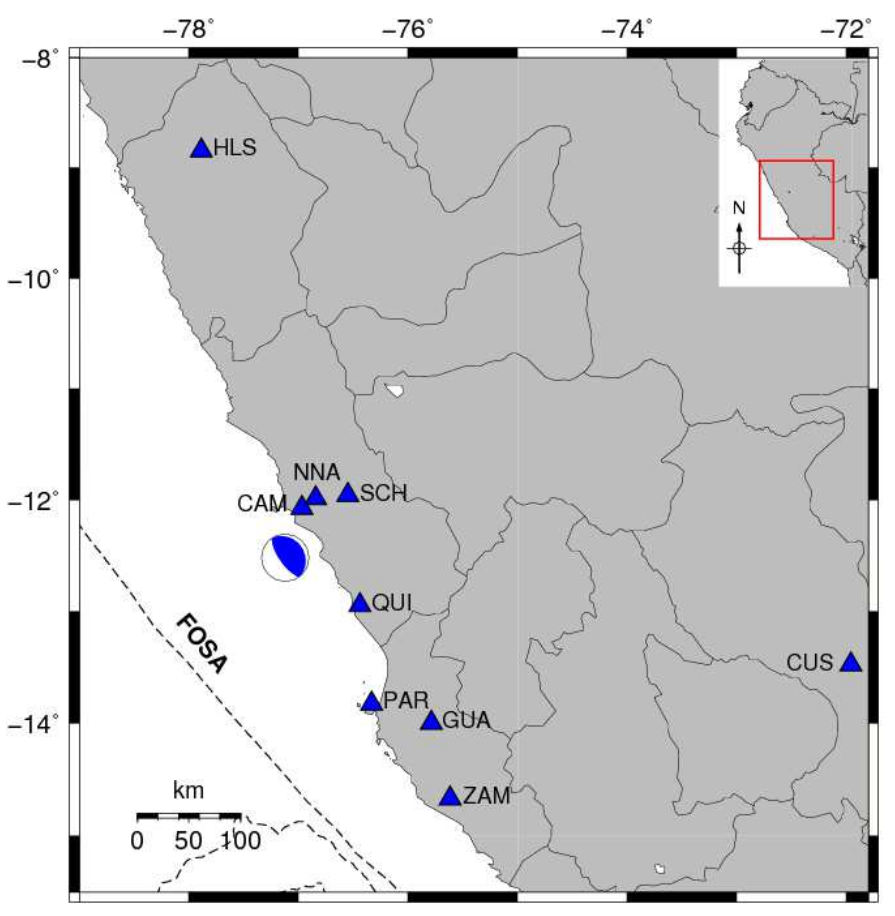

Figura 2: Ubicación del epicentro (esfera focal azul). Las estaciones sísmicas están representadas por los triángulos de color azul. Nótese la falta de estaciones sísmicas hacia el lado del mar. 
incógnitas son los 4 incrementos $(\delta t, \delta x, \delta y, \delta h)$. En forma matricial:

$$
G_{i j} \delta x j=r_{i}, i=1, \ldots, N, j=1, \ldots, 4
$$

Donde: $G_{i j}$ es la matriz de derivadas parciales y $\delta x_{j}$ (vertor del modelo $m_{j}$ son los incrementos de los parámetros hipocentrales. La solución es aquella para la cual la suma de los cuadrados de los residuales es mínima:

$$
S=\sum_{i=l_{i}}^{N} r_{i}^{2} \rightarrow 0
$$

Luego los incrementos obtenidos se suman a los valores iniciales y se repite el proceso en forma iterativa hasta obtener un residual aceptable (Udías, 1997).

\section{El método de inversión generalizada}

De la ecuación linealizada (5) y haciendo un cambio de variable $\delta x$ por $\mathrm{m}$ y $r_{i}$ por d, multiplicando por la traspuesta de $G\left(G^{T}\right)$ a cada miembro, se obtiene:

$$
\left(G^{T} G\right) m=G^{T} d
$$

Esta una forma muy útil llamada "ecuaciones normales". Ahora $G^{T} G$ es una matriz cuadrada, por lo que tiene inversa (siempre que no sea singular). Además $G^{T} G$ es una matriz simétrica, lo que significa que sus autovalores son reales y no-negativos. Por lo tanto, podemos escribir una ecuación de la forma:

$$
m=\left(G^{T} G\right)^{-1} G^{T} d
$$

Donde $\left(\left(G^{T} G\right)^{-1}\right) G^{T}=G^{-g}$ es llamada la inversa generalizada de G. Estrictamente hablando, esto es la inversa por mínimos cuadrados; si $G^{T} G$ es no-singular, entonces será la inversa generalizada. La ecuación (8) proporciona la mejor solución desde el punto de vista de los mínimos cuadrados, puesto que se minimiza el cuadrado del error. La ecuación (8) es una de las más importantes ecuaciones en Geofísica tanto para problemas lineales como no-lineales. En general, la mayoría de los problemas que se plantean son no-lineales. De este modo, $\mathrm{m}$ sólo servirá como una corrección del modelo inicial, por lo que se debe repetir el proceso de inversión varias veces, con G actualizado para el nuevo modelo (Lay y Wallace, 1995).

Para calcular la matriz de derivadas parciales $G$, se procede de la siguiente manera. Sea $t_{0}$ y $t_{i}$ el tiempo origen del sismo y el tiempo de arribo a la i-ésima estación, respectivamente. Entonces:

$$
t_{i}=t_{0}+\frac{\sqrt{\left(x_{i}-x\right)^{2}+\left(y_{i}-y\right)^{2}+\left(z_{i}-z\right)^{2}}}{v}
$$

Donde, $x_{i}, y_{i}, z_{i}$, son las coordenadas de las estaciones sísmicas; $x, y, z$ son las coordenadas del epicentro (las cuales convergen al valor real en cada iteración) y $v$ es la velocidad de la onda sísmica en el medio elástico homogéneo. Se define la matriz $\mathrm{G}$ de derivadas parciales como (Lay y Wallace, 1995):

$$
G_{i j}=\frac{\partial d_{i}}{\partial m_{j}}
$$

Donde $d_{i}$ representa a los datos observados, es decir, los tiempos de arribo $t_{i}$ y $m_{j}$ representa las incógnitas: $\mathrm{x}$, $y, z, t_{0}$. Ahora se puede determinar analíticamente la matriz G. En este caso, esto es relativamente simple puesto que las derivadas analíticas de la expresión (9) evaluadas en 0 son fáciles de obtener:

$$
\begin{gathered}
\frac{\partial t_{i}}{\partial x}=\frac{-\left(x_{i}-x_{0}\right)}{v^{2}\left(t_{i}-t_{0}\right)} \quad \frac{\partial t_{i}}{\partial y}=\frac{-\left(y_{i}-y_{0}\right)}{v^{2}\left(t_{i}-t_{0}\right)} \\
\frac{\partial t_{i}}{\partial z}=\frac{-\left(z_{i}-z_{0}\right)}{v^{2}\left(t_{i}-t_{0}\right)} \quad \frac{\partial t_{i}}{\partial t_{0}}=1
\end{gathered}
$$

Se ha implementado un código en Matlab que utiliza el algoritmo de la inversión generalizada para el cálculo de los parámetros hipocentrales. Los datos de entrada son: las lecturas de los tiempos de arribo de las fases $\mathrm{P}$ y $\mathrm{S}$, la ubicación de las estaciones sísmicas, la velocidad de la onda $P$ para un medio homogéneo y la condición inicial o hipocentro de prueba, el cual es ajustado en cada iteración.

\section{Datos}

Como ejemplo de aplicación se tomaron los datos disponibles del sismo del 28 de mayo de 2003 ocurrido a las 16:26 hora local (21:26 UTC), de magnitud 5.6 Mw, con epicentro a $42 \mathrm{~km}$ al oeste de la ciudad de Chilca, al sur de Lima (datos disponibles en: www.igp.gob.pe). Los datos de las lecturas de los tiempos de arribo corresponden a las estaciones de la Red Sísmica Nacional del Instituto Geofísico del Perú (IGP). Se han tomado las lecturas de 9 estaciones sísmicas ubicadas en el campo cercano, a una distancia epicentral no mayor a $600 \mathrm{~km}$ (Figura 2).

Las coordenadas de ubicación de las estaciones sísmicas están en el archivo estacion.txt que tiene el formato dado en la Tabla 2.

\section{Resultados}

Después de correr el modelo para 6 iteraciones, se obtuvieron los resultados dados en la Tabla 3. Como condición inicial, se tomó la posición de la estación sísmica más cercana (dada por el tiempo de arribo respectivo), luego el modelo realiza la inversión obteniendo un resultado que converge a la solución con cada iteración. El error medio cuadrático (rmse) es de $0.43 \mathrm{~s}$, un valor bastante aceptable, sabiendo que este parámetro no debe ser mayor a la unidad. 


\begin{tabular}{|l|c|c|c|r|}
\hline \multicolumn{5}{|c|}{28 May 2003 21h $26 \mathrm{~m}$} \\
\hline CAM & OEP & 58.80 & $0 \mathrm{ES}$ & 66.30 \\
\hline SCH & OEP & 63.40 & 0 & 0.00 \\
\hline QUI & OEP & 64.00 & 0 & 0.00 \\
\hline PAR & 0EP & 74.40 & 0 & 0.00 \\
\hline GUA & 0EP & 80.50 & 0 & 0.00 \\
\hline ZAM & OEP & 88.80 & 0 & 0.00 \\
\hline NNA & OEP & 60.70 & 0EP & 68.40 \\
\hline CUS & OEP & 128.10 & 0EP & 197.60 \\
\hline HLS & OEP & 106.30 & 0EP & 149.60 \\
\hline
\end{tabular}

Tabla 1: Tiempo de arribo de las fases sísmicas $\mathrm{P}$ y $\mathrm{S}$ (Fuente: IGP).

De acuerdo a la información de la Tabla 3, la estación sísmica más cercana es la de Camacho (CAM) con una distancia epicentral de $60 \mathrm{~km}$. La estación con un menor residual es Suche (SCH) y Cusco (CUS) con 0.0, mientras que la estación con un mayor residual es la de Zamaca (ZAM) con -0.9.

Si bien es cierto que el tiempo de arribo de la fase $S$ $\left(t_{s}\right)$ no interviene directamente en los cálculos del proceso de inversión; sin embargo, estas lecturas sirven para controlar el tiempo origen inicial $\left(t_{0}\right)$ del sismo mediante el método del diagrama de Wadati. Como mínimo se debe tener 2 lecturas de la fase S.

Para efectos de comparación, los parámetros hipocentrales calculados por el IGP, NEIC (National Earthquake Information Center) y este estudio están mostrados en la Tabla 4 y en la Figura 3. Se observa una buena distribución del epicentro con respecto a los calculados por el NEIC e IGP. La velocidad de la onda P asociada al menor residual es de $7.6 \mathrm{~km} / \mathrm{s}$, valor que se obtuvo al variar dicha velocidad durante el proceso de inversión (Figura 4).

\section{Discusión}

A continuación se analizará la matriz de autovectores del sistema $G^{T} G$, que está dada por $\mathrm{U}$, donde cada columna representa a un autovector:

$$
U=\left|\begin{array}{cccc}
0.1801 & 0.9454 & 0.2627 & -0.0689 \\
0.1440 & 0.2391 & -0.9602 & -0.0035 \\
0.9729 & -0.2092 & 0.0937 & 0.0295 \\
-0.0158 & 0.0724 & 0.0120 & 0.9972
\end{array}\right|
$$

Como el sistema $G^{T} G$ es una matriz simétrica, significa que sus autovalores son reales y no-negativos. La matriz de autovalores de $G^{T} G$ está dada por V:

$$
V=\left|\begin{array}{cccc}
0.0025 & 0 & 0 & 0 \\
0 & 0.0103 & 0 & 0 \\
0 & 0 & 0.0922 & 0 \\
0 & 0 & 0 & 9.0509
\end{array}\right|
$$

\begin{tabular}{|l|r|r|r|}
\hline Estación & Lat $\left(^{\circ}\right)$ & Lon $\left(^{\circ}\right)$ & Altitud $(\mathrm{m})$ \\
\hline CAM & -12.075 & -76.969 & 274 \\
\hline SCH & -11.960 & -76.549 & 2880 \\
\hline QUI & -12.946 & -76.439 & 600 \\
\hline PAR & -13.829 & -76.332 & 150 \\
\hline GUA & -13.998 & -75.790 & 690 \\
\hline ZAM & -14.670 & -75.615 & 390 \\
\hline NNA & -11.988 & -76.842 & 575 \\
\hline CUS & -13.478 & -71.959 & 3858 \\
\hline HLS & -08.847 & -77.889 & 3230 \\
\hline
\end{tabular}

Tabla 2: Código y coordenadas de las estaciones sísmicas.

Cada elemento de un autovector en particular corresponde a una dependencia del autovalor sobre un parámetro del modelo dado. Por ejemplo, el autovalor de mayor valor, 9.0509, está asociado con el autovector en la cuarta columna de U. Cada elemento de este vector está relacionado a un parámetro del modelo. El cuarto parámetro del modelo (0.9972), el cual está asociado al tiempo origen t0 domina a este autovector. El vector "apunta" en la dirección del tiempo origen $t_{0}$. Este autovalor es mucho mayor que los otros tres y por lo tanto, la estimación de este parámetro es más estable. Esto implica la importancia de las lecturas de las fases $\mathrm{S}\left(t_{s}\right)$, porque controlan la elección del valor inicial del tiempo origen, lo que implica un menor número de iteraciones en el proceso de inversión.

Por otro lado, el autovalor más pequeño (0.0025), con su autovector correspondiente (primera columna de U) está dominado por el tercer parámetro del modelo (0.9729), asociado con la profundidad focal z. De modo que la estimación de los cambios en la profundidad es la parte menos estable del proceso de inversión. Existe dificultad en determinar la verdadera profundidad a partir del valor inicial del modelo $\left(z_{0}=33 \mathrm{~km}\right)$, como se aprecia en la Tabla 5 , después de la primera iteración el valor diverge (a 94 $\mathrm{km}$ ) y luego de 5 iteraciones recién la profundidad focal se estabiliza, mientras que los otros parámetros (longitud y latitud) convergen rápidamente a la solución, incluso desde la primera iteración para el parámetro tiempo origen.

\section{Conclusiones}

Se ha implementado un programa computacional (en lenguaje de programación Matlab) para el cálculo de los parámetros hipocentrales mediante el algoritmo de inversión generalizada. Los resultados son bastante cercanos a los obtenidos por el IGP y por el NEIC.

De acuerdo a las condiciones iniciales: el tiempo origen inicial (51.8 s) se estimó a partir del diagrama de Wadati, la profundidad focal inicial se fijó en $33 \mathrm{~km}$ y las coordenadas iniciales del epicentro se toman de la estación sísmica más cercana. 


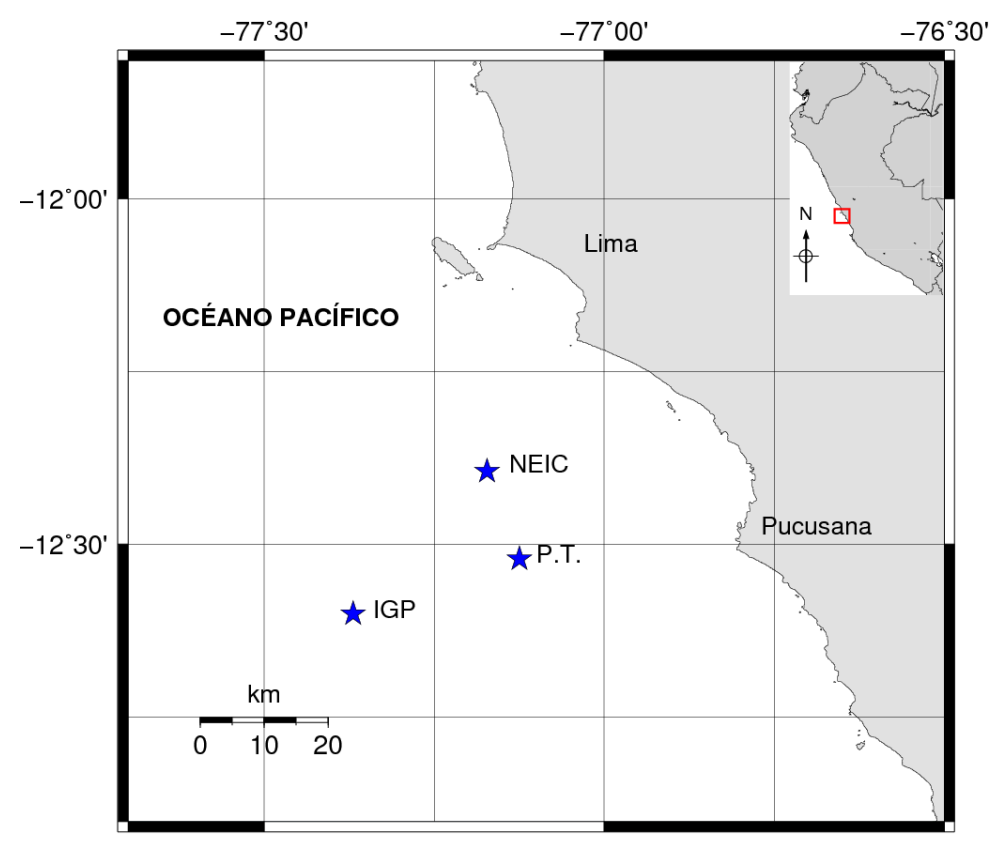

Figura 3: Epicentros calculados por el IGP, NEIC y el PT (presente trabajo) representados por las estrellas azules.

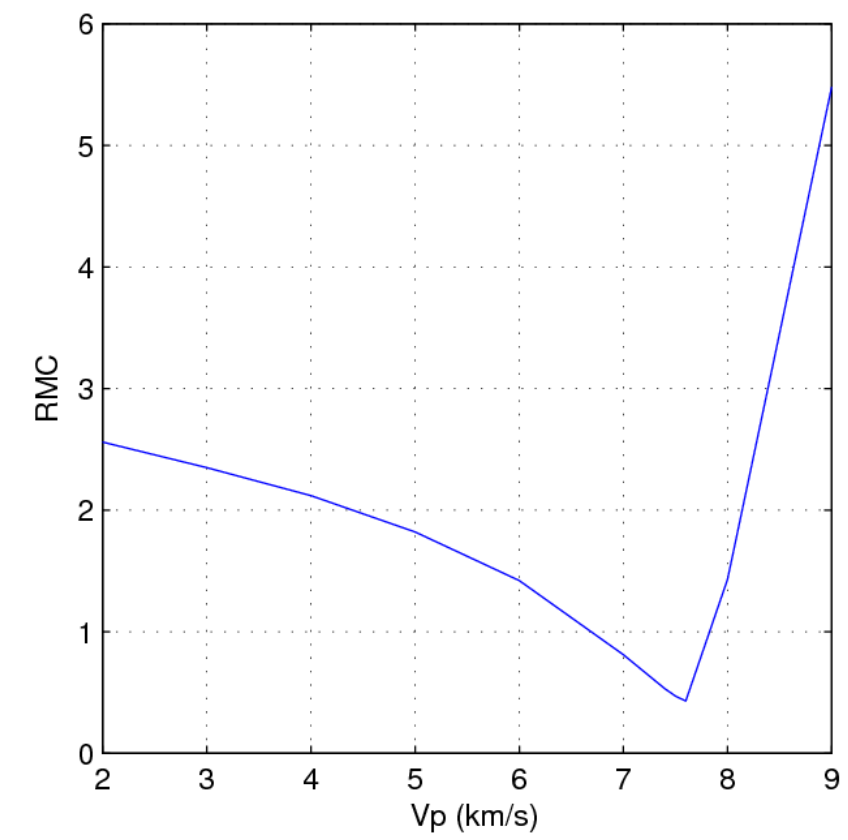

Figura 4: Gráfica Residual vs. Velocidad de la onda P. El residual mínimo es de $0.43 \mathrm{~s}$, que corresponde a una velocidad de la onda $P$ de $7.6 \mathrm{~km} / \mathrm{s}$. 


\begin{tabular}{|c|c|c|c|}
\hline \multicolumn{2}{|c|}{ Latitud } & \multicolumn{2}{|c|}{$-12.521^{\circ}$} \\
\hline \multicolumn{2}{|c|}{ Longitud } & \multicolumn{2}{|c|}{$-77.125^{\circ}$} \\
\hline \multicolumn{2}{|r|}{ Prof. } & \multicolumn{2}{|c|}{$30 \mathrm{~km}$} \\
\hline \multicolumn{2}{|c|}{ Tiempo } & \multicolumn{2}{|c|}{$21: 26: 51$} \\
\hline \multicolumn{2}{|r|}{ RMC } & \multicolumn{2}{|c|}{$0.43 \mathrm{~s}$} \\
\hline \multicolumn{2}{|c|}{ Iteración } & \multicolumn{2}{|c|}{6} \\
\hline $\mathrm{N}$ & Estación & $\operatorname{Res}(\mathrm{s})$ & $\Delta(\mathrm{km})$ \\
\hline 1 & CAM & -0.3 & 60.4 \\
\hline 2 & $\mathrm{SCH}$ & -0.0 & 93.4 \\
\hline 3 & QUI & 0.5 & 94.4 \\
\hline 4 & PAR & 0.6 & 172.7 \\
\hline 5 & GUA & 0.0 & 223.3 \\
\hline 6 & ZAM & -0.9 & 293.6 \\
\hline 7 & NNA & -0.1 & 73.3 \\
\hline 8 & CUS & 0.0 & 584.8 \\
\hline 9 & HLS & 0.2 & 418.1 \\
\hline
\end{tabular}

Tabla 3: Resultados del cálculo de los parámetros hipocentrales. $\Delta$ : distancia epicentral.

\begin{tabular}{|l|c|c|c|c|}
\hline Institución & Lat $\left(^{\circ}\right)$ & Lon $\left(^{\circ}\right)$ & Prof. $(\mathrm{km})$ & $t_{0}(\mathrm{~s})$ \\
\hline IGP & -12.600 & -77.369 & 20 & 44.8 \\
\hline NEIC & -12.394 & -77.172 & 43 & 47.3 \\
\hline Este estudio & -12.521 & -77.125 & 30 & 51.1 \\
\hline
\end{tabular}

Tabla 4: Comparación entre parámetros hipocentrales del IGP, NEIC y este estudio.

\begin{tabular}{|l|c|c|c|c|r|}
\hline $\mathrm{N}$ & 0 & 1 & 3 & 5 & 6 \\
\hline Lon $\left(^{\circ}\right)$ & -76.97 & -77.14 & -77.12 & -77.12 & -77.12 \\
\hline Lat $\left(^{\circ}\right)$ & -12.07 & -12.56 & -12.52 & -12.52 & -12.52 \\
\hline $\mathrm{z}(\mathrm{km})$ & 33.0 & 94.5 & 43.0 & 30.2 & 30.0 \\
\hline$t_{0}(\mathrm{~s})$ & 51.8 & 51.1 & 51.0 & 51.1 & 51.1 \\
\hline
\end{tabular}

Tabla 5: Comparación del hipocentro inicial (que corresponde a la ubicación de la estación más cercana y el tiempo origen dado por el diagrama de Wadati) y las soluciones después de $\mathrm{N}$ iteraciones. La iteración $\mathrm{N}=0$ representa las condiciones iniciales.

Se ha obtenido, a partir del proceso de inversión, una velocidad de la onda $\mathrm{P}$ de $7.6 \mathrm{~km} / \mathrm{s}$, considerando un modelo de velocidad homogéneo en un espacio semi-infinito. La relación entre las velocidades $\mathrm{P}$ y $\mathrm{S}$ es 1.87 .

De acuerdo al análisis de los autovectores y autovalores, el parámetro más estable es el tiempo origen (que converge rápidamente a la solución incluso después de la primera iteración), mientras que el parámetro menos estable es la profundidad focal (que se estabiliza después de varias iteraciones, en este caso la quinta iteración).

\section{Referencias}

[1] Bravo, J. (1996). Problema de la localización de terremotos. Instituto Geográfico Nacional, Madrid, España. pp 17-46.

[2] Havskov, J. y Ottemoller, L. (2010). Routine Data
Processing in Earthquake Seismology. University of Bergen, Norway. Springer Editorial.

[3] IGP (2015). Página web del Instituto Geofísico del Perú: www.igp.gob.pe

[4] Jiménez, C. (2010). Cálculo de la magnitud sísmica para la estación de Ñaña. Revista de Investigación de Física, Vol 13, N 1, 101301755.

[5] Klein, F. (2014). Hypoinverse 2000: Fortran program to solve for earthquake location and Magnitudes: http://earthquake.usgs.gov/research/software/

[6] Lahr, J.C., 1999, revised 2012, Hypoellipse: a computer program for determining local earthquake hypocentral parameters, magnitude, and firstmotion pattern: USGS Open-File Report 99-23, 
version $1.1,119$ p. and software, available at: http://pubs.usgs.gov/of/1999/ofr-99-0023/

[7] Lay, T. and Wallace, T. (1995). Modern Global Seismology. Academic Press, Inc. 1st Edition. California.

[8] Norabuena, E., Leffler, L. Mao, A. Dixon, T., Stein, S., Saks, S., Ocola, L., Ellis, M. (1998). Space Geodetic Observations of Nazca-South America Convergence across the Central Andes; Science 279, 358
[9] Udías, A. y Mezcua, J. (1997). Fundamentos de Sismología, 1ra Edición. UCA Editores. El Salvador.

[10] Tarantola, A. (2005). Inverse Problem Theory and Methods for Model Parameter Estimation. SIAM: Society for Industrial and Applied Mathematics. Paris, France. 